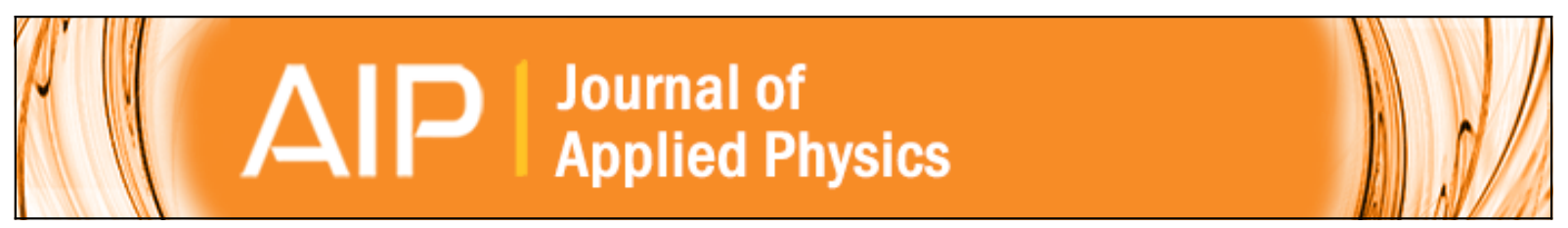

\title{
Optical constants of SrF2 thin films in the $25-780-e V$ spectral range
}

Luis Rodríguez-de Marcos, Juan I. Larruquert, José A. Aznárez, Mónica Fernández-Perea, Regina Soufli, José

A. Méndez, Sherry L. Baker, and Eric M. Gullikson

Citation: Journal of Applied Physics 113, 143501 (2013); doi: 10.1063/1.4800099

View online: http://dx.doi.org/10.1063/1.4800099

View Table of Contents: http://scitation.aip.org/content/aip/journal/jap/113/14?ver=pdfcov

Published by the AIP Publishing

\section{Articles you may be interested in}

Transmittance and optical constants of Sr films in the 6-1220 eV spectral range

J. Appl. Phys. 111, 113533 (2012); 10.1063/1.4729487

Transmittance and optical constants of Ho films in the 3-1340 eV spectral range

J. Appl. Phys. 109, 083525 (2011); 10.1063/1.3556451

Transmittance and optical constants of Lu films in the 3-1800 eV spectral range

J. Appl. Phys. 108, 063514 (2010); 10.1063/1.3481062

Optical constants of evaporation-deposited silicon monoxide films in the 7.1-800 eV photon energy range

J. Appl. Phys. 105, 113505 (2009); 10.1063/1.3123768

Transmittance and optical constants of Tm films in the $2.75-1600 \mathrm{eV}$ spectral range

J. Appl. Phys. 105, 103110 (2009); 10.1063/1.3129507

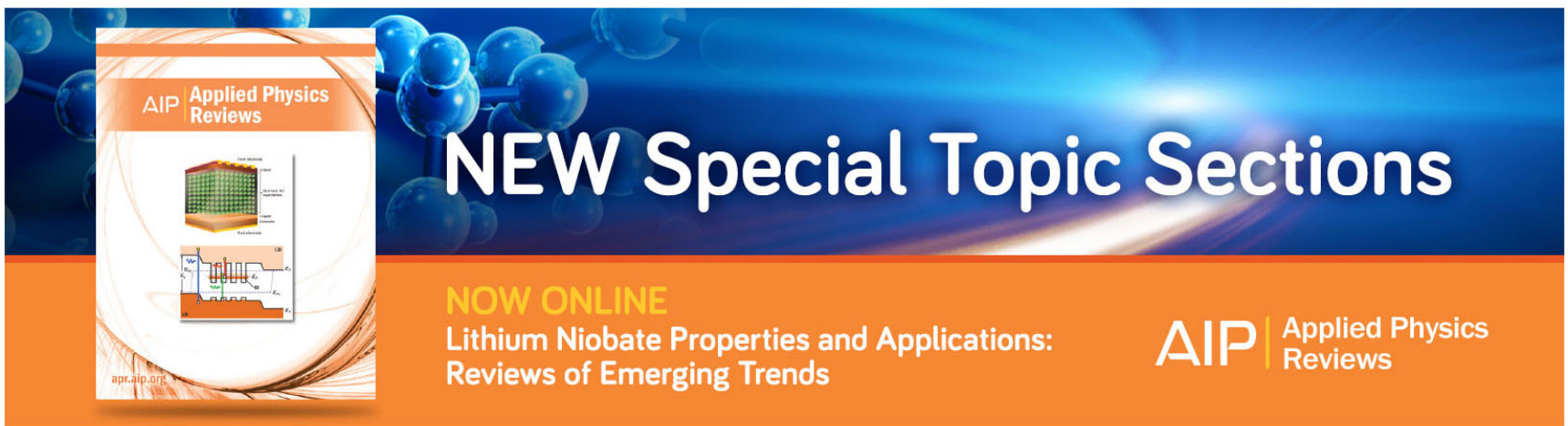




\title{
Optical constants of $\mathrm{SrF}_{\mathbf{2}}$ thin films in the $25-780-\mathrm{eV}$ spectral range
}

\author{
Luis Rodríguez-de Marcos, ${ }^{1}$ Juan I. Larruquert, ${ }^{1, a)}$ José A. Aznárez, ${ }^{1}$ \\ Mónica Fernández-Perea, ${ }^{2}$ Regina Soufli, ${ }^{2}$ José A. Méndez, ${ }^{1}$ Sherry L. Baker, ${ }^{2}$ \\ and Eric M. Gullikson ${ }^{3}$ \\ ${ }^{1}$ GOLD-Instituto de Óptica-Consejo Superior de Investigaciones Científicas, Serrano 144, 28006 Madrid, \\ Spain \\ ${ }^{2}$ Lawrence Livermore National Laboratory, 7000 East Avenue, Livermore, California 94550, USA \\ ${ }^{3}$ Lawrence Berkeley National Laboratory, 1 Cyclotron Road, Berkeley, California 94720, USA
}

(Received 21 February 2013; accepted 21 March 2013; published online 8 April 2013)

\begin{abstract}
The transmittance and the optical constants of $\mathrm{SrF}_{2}$ thin films, a candidate material for multilayer coatings operating in the extreme ultraviolet and soft x-rays, have been determined in the spectral range of $25-780 \mathrm{eV}$, in most of which no experimental data were previously available. $\mathrm{SrF}_{2}$ films of various thicknesses were deposited by evaporation onto room-temperature, thin $\mathrm{Al}$ support films, and their transmittance was measured with synchrotron radiation. The transmittance as a function of film thickness was used to calculate the extinction coefficient $\mathrm{k}$ at each photon energy. A decrease in density with increasing $\mathrm{SrF}_{2}$ film thickness was observed. In the calculation of $\mathrm{k}$, this effect was circumvented by fitting the transmittance versus the product of thickness and density. The real part of the refractive index of $\mathrm{SrF}_{2}$ films was calculated from $\mathrm{k}$ with Kramers-Krönig analysis, for which the measured spectral range was extended both to lower and to higher photon energies with data in the literature combined with interpolations and extrapolations. With the application of $\mathrm{f}$ - and inertial sum rules, the consistency of the compiled data was found to be excellent. (C) 2013 American Institute of Physics. [http://dx.doi.org/10.1063/1.4800099]
\end{abstract}

\section{INTRODUCTION}

The demand for novel multilayer coatings for the extreme ultraviolet (EUV) and soft x-rays (SXR) ranges is rising since they are required for many applications, such as EUV lithography, tabletop and free-electron lasers, space instrumentation for astrophysics and solar physics, synchrotron radiation, etc. In order to design multilayer coatings, it is necessary to use accurate optical constants of suitable materials, such as materials with low absorption. $\mathrm{SrF}_{2}$ is a material for which a low absorption band has been reported in a portion of the EUV range. ${ }^{1}$ This paper addresses the optical characterization of $\mathrm{SrF}_{2}$ thin films in the EUV and SXR ranges. Few data have been reported in the literature on thin films of $\mathrm{SrF}_{2}$. Lukirskii et al. ${ }^{2}$ measured the reflectance versus angle of thin films of $\mathrm{SrF}_{2}$ and many other materials at 5 photon energies between 110 and $525 \mathrm{eV}$, from which they calculated the optical constants: the refractive index decrement $\delta=1-\mathrm{n}$ and the extinction coefficient k. RobinKandare and Robin $^{3}$ measured the transmittance of $\mathrm{SrF}_{2}$ (along with $\mathrm{CaF}_{2}$ and $\mathrm{BaF}_{2}$ ) thin films, from which they calculated both absorption (neglecting reflectance) and the absorption coefficient in the $\sim 9-14-\mathrm{eV}$ range; additionally, they measured reflectance for cleaved monocrystals of these materials in the 9.5-13.8-eV range. Finally, Frandon et al. ${ }^{4}$ performed electron energy loss spectroscopy on $\mathrm{SrF}_{2}$ films and calculated their complex dielectric constant in the 5-35-eV range. The scant available data for $\mathrm{SrF}_{2}$ films do not fully cover the EUV-SXR spectral ranges. The optical

\footnotetext{
a) Author to whom correspondence should be addressed. Electronic mail: larruquert@io.cfmac.csic.es. Fax: 34914117651.
}

constants of $\mathrm{SrF}_{2}$ up to $35 \mathrm{eV}$ were reviewed and tabulated by Thomas; ${ }^{5}$ the review was focused on optical constants measured on bulk $\mathrm{SrF}_{2}$, such as crystals. Thin films often grow with lower density, amorphous or nanocrystalline structure, and larger content of defects and voids compared to bulk crystals. Dielectric films, particularly some fluorides, deposited on substrates at room temperature grow with considerable porosity, ${ }^{6,7}$ which results in a reduced density compared to crystals. This may result in that the optical constants of films and bulk crystals of the same compound are largely different, particularly in the transparent region, in which the thin film presents a high loss compared to the crystal, as it has been shown for $\mathrm{MgF}_{2} \cdot{ }^{8,9}$ Furthermore, $\mathrm{SrF}_{2}$ films have been reported to grow nonuniform in depth (Valeev, ${ }^{10}$ as cited by Gisin ${ }^{11}$ ). El-Shazly and Ebrahim ${ }^{12}$ found that $\mathrm{SrF}_{2}$ films deposited by evaporation on substrates not hotter than $50{ }^{\circ} \mathrm{C}$ were inhomogeneous in depth and they determined two different refractive indices in the 460-1000-nm spectral range: a lower one for the film layer adjacent to the air and a larger one for the film layer adjacent to the glass substrate. Gisin ${ }^{11}$ reported the dependence of the refractive index of $\mathrm{SrF}_{2}$ thin films on film thickness at the wavelength of $4.5 \mu \mathrm{m}$ for films deposited at $25^{\circ} \mathrm{C}$; the refractive index continuously decreased over a range of thicknesses between 0.8 and $7.4 \mu \mathrm{m}$. Therefore, the optical properties of $\mathrm{SrF}_{2}$ films are expected to not only differ from those of the bulk material but to depend on the film thickness.

This paper reports on the optical properties of $\mathrm{SrF}_{2}$ thin films in the EUV-SXR ranges. Given that in many applications, it is desirable not to heat the substrate upon thin-film deposition, the films were deposited by evaporation onto roomtemperature substrates. Section II describes the experimental 
techniques used in the preparation of the $\mathrm{SrF}_{2}$ samples and in their characterization. Section III reports the transmittance of $\mathrm{SrF}_{2}$ films of various thicknesses in the 25-780-eV range. Transmittance measurements as a function of film thickness are used to calculate $\mathrm{k}$ in the same range; an extrapolation of $\mathrm{k}$ to lower and higher photon energies using data from the literature and models is used to calculate $\mathrm{n}$, the real part of the refractive index, with Kramers-Krönig (KK) analysis. The consistency of the determined values of $\mathrm{n}$ and $\mathrm{k}$ is also examined.

\section{EXPERIMENTAL TECHNIQUES}

\section{A. Sample preparation}

$\mathrm{SrF}_{2}$ films were deposited by evaporation of offcuts of vacuum-ultraviolet-grade $\mathrm{SrF}_{2}$ crystals; tungsten boats were used as resistive sources. In the deposition chamber, the base pressure and the pressure during deposition were $\sim 2 \cdot 10^{-5}$ and $10^{-4} \mathrm{~Pa}$, respectively; the chamber was evacuated with a turbo pump system. The deposition rate was $0.5 \mathrm{~nm} / \mathrm{s}$. The substrates were not intentionally heated or cooled during deposition. The sample holder had four $12.5 \times 12.5 \mathrm{~mm}^{2}$ quadrants inside a $40 \times 40 \mathrm{~mm}^{2}$ square. Two quadrants held each a multiple thin-film substrate for transmittance measurements; another quadrant held a Si substrate; and the remaining quadrant held a glass substrate. The Si substrate, used for reflectance measurements at $13.5 \mathrm{~nm}$, was a piece cut from a $\mathrm{Si}$ wafer. The glass substrate was a piece of polished float glass; it was used for thickness measurements with Tolansky interferometry, i.e., through multiple-beam interference fringes in a wedge between two highly reflective surfaces. $^{13}$

The thin-film substrates consisted of unbacked, 25-nm thick Al films supported on a $0.2-\mathrm{mm}$ thick, perforated $\mathrm{Cu}$ plate. On each $\mathrm{Cu}$ plate, we drilled five $1.5-\mathrm{mm}$ diameter holes to place the Al-film substrates. To produce the unbacked Al films, a collodion film was previously cast on the perforated $\mathrm{Cu}$ plates and the $\mathrm{Al}$ film was then vacuumdeposited on the collodion-coated plates. Finally, the collodion film was thoroughly dissolved leaving the unbacked $\mathrm{Al}$ film substrate over each hole for EUV transmittance measurements. During $\mathrm{SrF}_{2}$ evaporation, two $\mathrm{Al}$ film substrates per $\mathrm{Cu}$ plate were masked and the other three were not. In that way, we could measure the transmittance of both the uncoated as well as the $\mathrm{SrF}_{2}$-coated $\mathrm{Al}$ film substrates for normalization purposes. The EUV transmittance was measured $\sim 4$ weeks after deposition.

\section{B. Experimental setup for transmittance measurements}

The EUV/SXR reflectance and transmittance measurements were performed at beamline 6.3.2. of the Advanced Light Source (ALS) synchrotron at Lawrence Berkeley National Laboratory (LBNL). The general characteristics of the beamline have been described in detail earlier. ${ }^{14,15}$ The sample chamber allows translation of the sample in three dimensions, tilt in two dimensions, and azimuth rotation of the sample holder. The available detectors include photodiodes and a CCD camera (the latter for sample alignment), which can be rotated $360^{\circ}$ around the axis of the chamber.

For the transmittance measurements, four monochromator gratings $(80,200,600$, and 1200 lines $/ \mathrm{mm})$ were used to access the photon energy range from $25 \mathrm{eV}$ to $780 \mathrm{eV}$. The monochromator exit slit was set to a width of $40 \mu \mathrm{m}$. Photon energy calibration was based on the absorption edges of a series of transmission filters $(\mathrm{Al}, \mathrm{Si}, \mathrm{Ti}$, and $\mathrm{Cr})$ with a relative accuracy of $0.011 \%$ RMS and with $0.007 \%$ repeatability. During the measurements, 2nd harmonic and stray light suppression was also achieved with a series of filters $(\mathrm{Mg}, \mathrm{Al}$, $\mathrm{Si}, \mathrm{Be}, \mathrm{B}, \mathrm{C}, \mathrm{Ti}, \mathrm{Cr}$, and $\mathrm{Co}$ ). For suppression of higher-order harmonics, an "order suppressor" consisting of three mirrors at a variable grazing incidence angle (depending on energy range) and based on the principle of total external reflection was used in addition to the filters. The ALS storage ring current was used to normalize the signal against the storage ring current decay. The base pressure in the measurement chamber was in the range $1.3 \times 10^{-4}-1.3 \times 10^{-5} \mathrm{~Pa}$. The signal was collected on a GaAsP photodiode detector with acceptance angle of $1^{\circ}$. The reflectance measurements at $91.8 \mathrm{eV}$ were obtained with the 200 lines/mm grating, a Be filter for 2nd-harmonic suppression, the order suppressor consisting of three carbon mirrors at $12^{\circ}$ grazing angle of incidence, and the GaAsP photodiode detector.

Atomic Force Microscopy (AFM) measurements were performed with a Digital Instruments Dimension $5000^{\mathrm{TM}}$ instrument equipped with an acoustic hood and vibration isolation, reaching a noise level of $0.03 \mathrm{~nm} \mathrm{rms}$. The instrument is operated in tapping mode, which measures topography in air by tapping the surface with an oscillating probe tip. The probe tips were etched silicon, with a nominal tip radius of $5-10 \mathrm{~nm}$.

\section{RESULTS AND DISCUSSION}

\section{A. Transmittance and extinction coefficient of $\mathrm{SrF}_{2}$}

The transmittance of $\mathrm{SrF}_{2}$ films with three different thicknesses $(20.0,46.0$, and $89.5 \mathrm{~nm}$ as obtained from Tolansky interferometry) was measured in the $25-780-\mathrm{eV}$ range. For normalization purposes, we also measured the transmittance of $\mathrm{Al}$ substrates that were prepared in the same runs as the substrates used to support the $\mathrm{SrF}_{2}$ films. Fig. 1 displays the transmittance of the three $\mathrm{SrF}_{2}$ films normalized to the transmittance of each substrate. Transmittance displays a deep minimum centered around $30 \mathrm{eV}$, which may be assigned to the $\mathrm{Sr} \mathrm{N}_{2,3}$ edge; the broad minimum centered at $\sim 220 \mathrm{eV}$ is also attributed to Sr; the oscillations at $\sim 270$ and $\sim 290 \mathrm{eV}$ are assigned to the $\mathrm{Sr} \mathrm{M}_{3}$ and $\mathrm{M}_{2}$ edges, respectively. ${ }^{16}$ The structure at $\sim 690 \mathrm{eV}$ and above is attributed to the $\mathrm{F} \mathrm{K}$ edge.

In the assumption that multiple reflections in the film are negligible, transmittance data versus film thickness can be used to calculate $\mathrm{k}$ with the well-known Beer-Lambert law

$$
\left(\frac{\mathrm{T}_{\mathrm{s}+\mathrm{f}}}{\mathrm{T}_{\mathrm{s}}}\right)_{\mathrm{i}}=\exp \left(-\frac{4 \pi \mathrm{kx}_{\mathrm{i}}}{\lambda}\right),
$$

where $x_{i}, i=1,2,3$, stands for the thickness of each of the $\mathrm{SrF}_{2}$ films; $\mathrm{T}_{\mathrm{s}}$ and $\mathrm{T}_{\mathrm{s}+\mathrm{f}}$ stand for the transmittance of the 


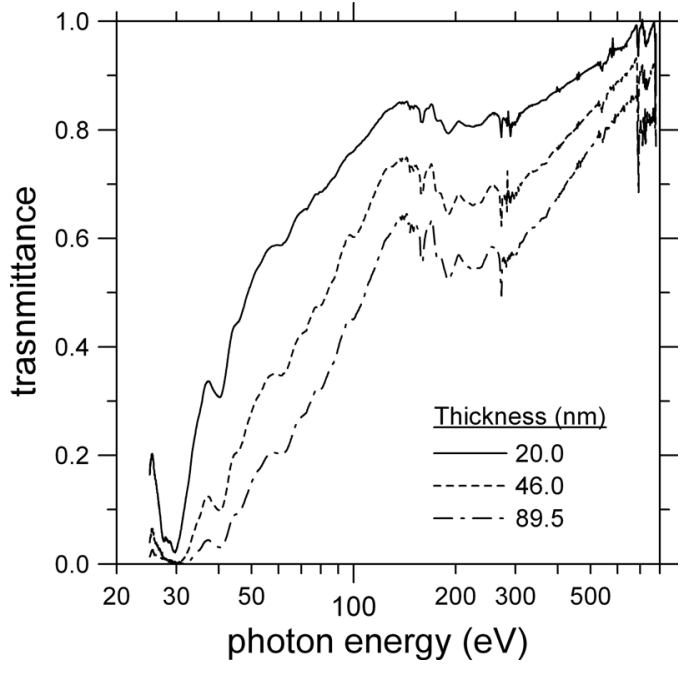

FIG. 1. The transmittance of three $\mathrm{SrF}_{2}$ films normalized to the transmittance of the substrate versus the photon energy in log scale.

substrate and of the substrate coated with $\mathrm{SrF}_{2}$, respectively, at wavelength $\lambda$. Photon energy and wavelength are related through $\mathrm{E}(\mathrm{eV})=1239.8 / \lambda(\mathrm{nm})$. From Eq. (1), a linear fit of the logarithm of the normalized transmittance data versus film thickness provides $\mathrm{k}$ at each wavelength. We will refer to this procedure as the slope method for $\mathrm{k}$ determination. Fig. 2 displays the normalized transmittance (in log scale) versus thickness for a selection of photon energies.

At each photon energy, the transmittance data do not fall in a straight line, and the decrease trend is less pronounced than what is predicted by Eq. (1). Furthermore, we observed a deviation between the film thickness measured with the quartz crystal monitor in the deposition chamber, which measures mass accumulated on the crystal, and the thickness measured by Tolanski interferometry, which measures real physical thickness. Such deviation, which was more pronounced for thicker films, indicated that the real film thickness was larger than the thickness expected from the deposited mass, and that the difference was increasing with film thickness. Both observations above are consistent with a

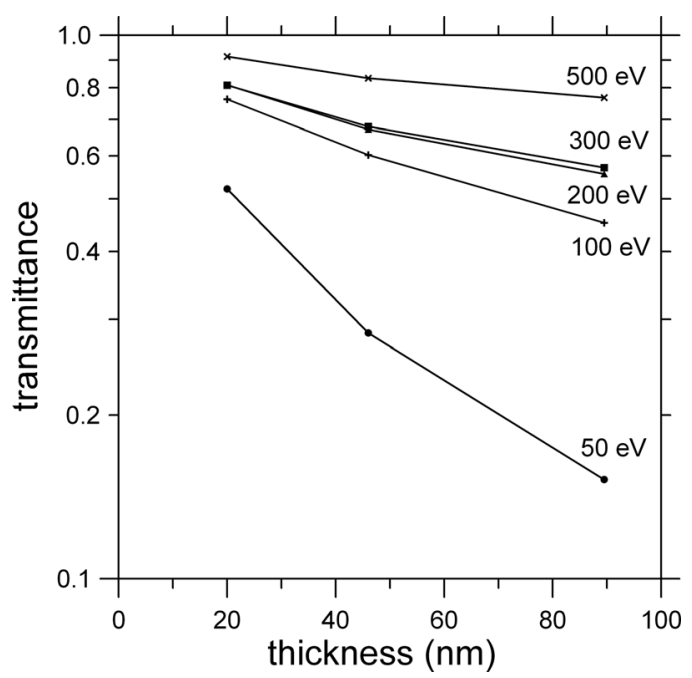

FIG. 2. The transmittance (log scale) of $\mathrm{SrF}_{2}$ films at five selected photon energies versus the film thickness. progressive decrease of film density with increasing $\mathrm{SrF}_{2}$ thickness, which is also compatible with the dependence of $\mathrm{SrF}_{2}$ optical constants with thickness reported in Refs. 11 and 12. In those references, this effect was observed for thicknesses larger than the present ones; density dependence with thickness is observed here at thicknesses as small as tens of nm.

Reflectance was measured as a function of the angle of incidence at $13.5 \mathrm{~nm}(91.8 \mathrm{eV})$ on three $\mathrm{Si}$ witness samples that had been coated in the same run that the $\mathrm{SrF}_{2}$ samples whose transmittance was measured. These measurements were attempted to fit using the IMD software. ${ }^{17}$ However, the fits obtained were not accurate enough as to obtain unambiguously the film density and the RMS roughness values of the interfaces. We believe that this was due to the unknown density gradient in depth and hence the optical-constant gradient through the films, which could not be successfully modeled.

In view of this, we calculated the average density of each $\mathrm{SrF}_{2}$ film using transmittance measurements in the following way. We compared our experimental transmittance with calculated values in which the density was left as a free parameter. At photon energies larger than $\sim 30 \mathrm{eV}$ and away from absorption edges, the optical properties of a material can be obtained to a good approximation by summing the effects of the individual atoms as if they were independent of each other, because the response of a material is mostly determined by tightly bound electrons, which are little affected by molecular binding. ${ }^{18}$ At photon energies in the EUV and SXR, the interaction of radiation with a single atom is given in terms of the atomic forward scattering factors. ${ }^{19}$ Optical constants of $\mathrm{SrF}_{2}$ for various densities were calculated in this approach using the available data on $\mathrm{Sr}$ and $\mathrm{F}$. For $\mathrm{Sr}$, we used the experimental characterization performed by Rodríguez-de Marcos et al. ${ }^{16}$ For $\mathrm{F}$, we used the semiempirical data of Henke et al., ${ }^{19}$ downloaded from the web of the Center for X-Ray Optics (CXRO) ${ }^{20}$ For each density, the contribution of $\mathrm{Sr}$ and $\mathrm{F}$ to $\mathrm{k}$ was weighted according to the number of atoms per unit volume and then summed up for the two species. For each film, we varied the density until the best match between calculated and experimental transmittance values was obtained; the fitted density was interpreted as the average film density. Fig. 3 shows the fits obtained for the three films; the fitted range was limited to $70-600 \mathrm{eV}$, in order to avoid both low photon energies and the F K edge, where calculations with the independent-atom approximation are expected to be less accurate; the low-energy limit was increased to $70 \mathrm{eV}$ to enable a more accurate fit. The fitted densities are displayed in Table I; we observe a dramatic density decrease versus increasing thickness in the present thickness range. The displayed thickness values were measured by using Tolansky interferometry.

AFM was used to measure the topography of the films in order to calculate the high spatial frequency roughness (HSFR) of the surface. The results are shown in Table I. Surface HSFR was computed from the Power Spectrum Density (PSD) in the spatial frequency range from $5 \times 10^{-4}$ to $0.05 \mathrm{~nm}^{-1}$, which is most relevant for reflectance in the EUV. The HSFR measurement of the $20.0 \mathrm{~nm}$-thick sample is expected to be accurate. The HSFR obtained for the 46.0-nm-thick film is expected to 


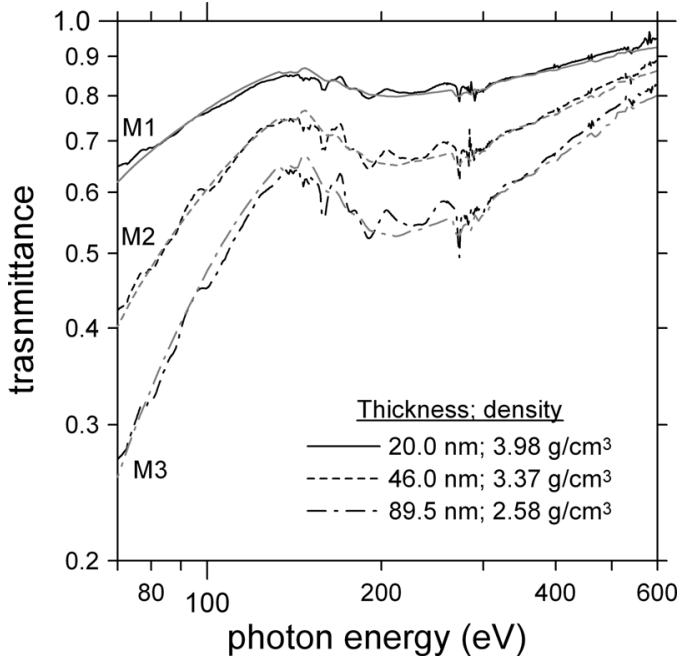

FIG. 3. Log-log plot of transmittance of three $\mathrm{SrF}_{2}$ films versus photon energy. Black lines: measurements. Grey lines: best fits, with density as the fitted parameter.

be underestimated, as it is likely that the real HSFR of this sample exceeded the upper limit of roughness that the AFM instrument is able to measure. The $89.5 \mathrm{~nm}$-thick sample could not be measured with AFM due to excessive roughness. The increase of roughness with thickness, along with the decrease of density, indicates an increase of the film porosity with thickness.

The dependence of $\mathrm{SrF}_{2}$ density on thickness means that the optical constants of $\mathrm{SrF}_{2}$ must also vary with thickness. In the independent-atom approximation, $\mathrm{k}$ is proportional to density $(\rho)$. We modified Eq. (1) to leave it as a function of $\mathrm{k}$ and $\rho$

$$
\left(\frac{T_{s+f}}{T_{s}}\right)_{i}=\exp \left[-\frac{4 \pi k_{\rho} \rho_{i} x_{i}}{\lambda}\right]
$$

where $\mathrm{k}_{\rho}$ stands for the extinction coefficient per unit density: $\mathrm{k}_{\rho}=\mathrm{k} / \rho$. Hence, $\mathrm{k}_{\rho}$ is independent of the specific density of each film, and transmittance depends on the product of thickness and density. Fig. 4 displays the normalized transmittance (in $\log$ scale) versus thickness $\times$ density for the same selection of photon energies of Fig. 2; densities were used as per Table I. In this way, we obtained satisfactory linear fits.

Using the transmittance data plotted in Fig. 1, and the thickness and density data displayed in Table I, we can obtain

TABLE I. Film thickness, average density, and HSFR of three SrF2 films. Thickness was determined with Tolansky interferometry. Density was obtained by fitting EUV transmittance measurements; bulk density is given for comparison. HSFR was obtained from AFM measurements in the $5 \times 10^{-4}$ to $0.05 \mathrm{~nm}^{-1}$ frequency range; the HSFR measurement in parentheses is considered an under-estimated value.

\begin{tabular}{lccc}
\hline \hline Sample & Film thickness (nm) & Density $\left(\mathrm{g} / \mathrm{cm}^{3}\right)$ & HSFR (nm RMS) \\
\hline M1 & 20.0 & 3.98 & 2.3 \\
M2 & 46.0 & 3.37 & $(3.3)$ \\
M3 & 89.5 & 2.58 & N/A \\
& Bulk & 4.28 & \\
\hline \hline
\end{tabular}

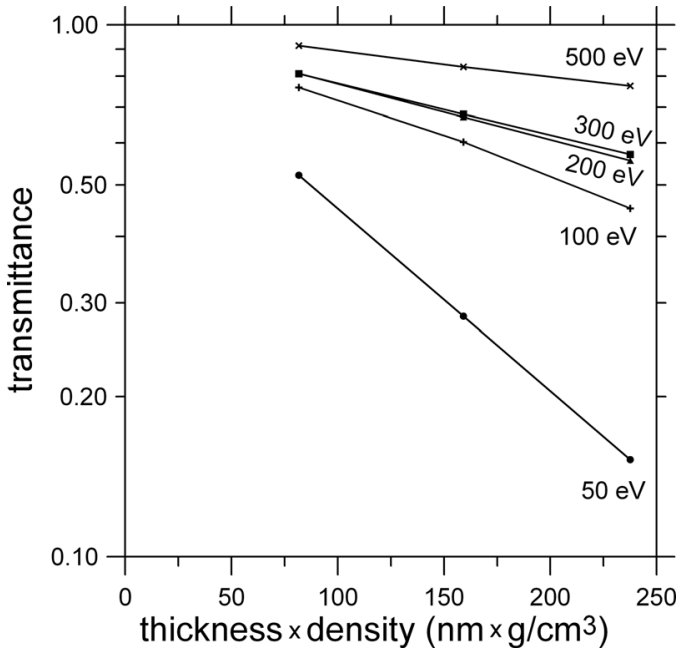

FIG. 4. The transmittance (log scale) of $\mathrm{SrF}_{2}$ films at five selected photon energies versus the product of film thickness $\times$ density.

$\mathrm{k}_{\rho}$ with Eq. (2). In the independent-atom approximation, $\mathrm{k}$ for each specific film density is obtained by multiplying $\mathrm{k}_{\rho}$ by the density. In the following, we will focus on the density of the thinnest sample $(20 \mathrm{~nm}, \mathrm{M} 1)$ because this film thickness is closer to the range of suitable values for multilayer coatings in the EUV/SXR range. Hence, in Fig. 5, we plot $\mathrm{k}=\mathrm{k}_{\rho} \rho_{1}$ versus photon energy. In the calculation of $\mathrm{k}$, we attempted to use the data from all 3 samples at each photon energy. However, in the ranges $25-32$ and $560-780 \mathrm{eV}$, the data were not consistent across the 3 samples. At $25-32 \mathrm{eV}$, the transmittance of samples M2 and M3 was larger than what would be expected from the transmittance of sample M1. This could be attributed to the large absorption of $\mathrm{SrF}_{2}$ in this range; such excessively high transmittance of M2 and M3 may be due to either low photon statistics or to the presence of pinholes in the films. Therefore, only data from M1 were used at $25-32 \mathrm{eV}$. When only data of one sample are available, one cannot calculate $\mathrm{k}$ with the aforementioned slope method; instead, we used Eq. (2) directly to obtain $\mathrm{k}$ from a single transmittance measurement. In the $560-780-\mathrm{eV}$ range, only the data from the M3 sample look

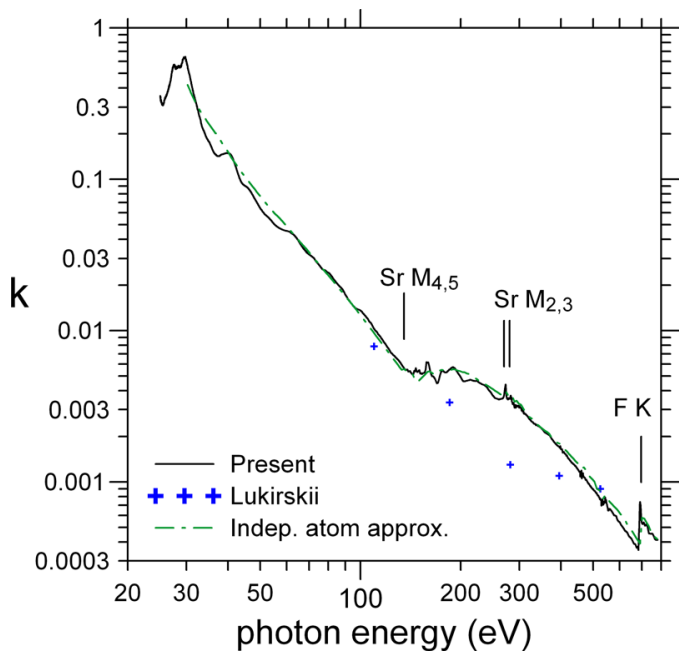

FIG. 5. Log-log plot of $\mathrm{k}$ of $\mathrm{SrF}_{2}$ films versus photon energy for a density of $3.98 \mathrm{~g} / \mathrm{cm}^{3}$. The data of Lukirskii $e t \mathrm{al}^{2}$ and the data calculated in the independent-atom approximation are also plotted. 
plausible. This was attributed to the very low absorption of the two thinnest films, resulting in reduced accuracy in the absorption determination. Hence, at $560-780 \mathrm{eV}$, only data from M3 were used. Fig. 5 also shows the data calculated with the independent-atom approximation, based on the experimental data on $\mathrm{Sr}^{16}$ and the semi-empirical data on $\mathrm{F}^{19,20} \mathrm{~A}$ good agreement is obtained, particularly in the $60-400-\mathrm{eV}$ range. The data of Lukirskii et al. ${ }^{2}$ are also plotted; a good agreement is not obtained with the latter data. The optical constants in Ref. 2. were calculated from reflectance measurements versus incidence angle; the roughness of the sample, which plays a much larger role in reflectance than in transmittance measurements, seems to not have been accounted for in the calculation, which might explain the poor agreement. Reflectance measurements are also more sensitive to surface contamination than transmittance measurements; in the latter, a contamination layer common to all measured films is mostly cancelled out when calculating $\mathrm{k}$ with the slope method. $\mathrm{k}$ at low photon energies is plotted in Fig. 6, along with data in the literature. The data of Nisar and Robin ${ }^{1}$ were obtained from reflectance measurements on single crystals of $\mathrm{SrF}_{2}$ that were cleaved in situ. They performed KK analysis on the reflectance data and obtained $\mathrm{n}$ and $\mathrm{k}$ in the $10-36-\mathrm{eV}$ range. They obtained a minimum value of $\mathrm{k}$ of $\sim 0.03$ at $26.9 \mathrm{eV}$, a photon energy close to the Ne-like Ar line laser operating at $26.4 \mathrm{eV}^{21}$ These reported low-absorption values were a motivation for this research, since, if verified, they could render $\mathrm{SrF}_{2}$ a promising material for multilayer coatings operating near $26.9 \mathrm{eV}$. Nevertheless, a much larger $\mathrm{k}$ value of 0.45 for $\mathrm{SrF}_{2}$ films was measured at $26.9 \mathrm{eV}$ in the present research. The present results at low photon energies are not far from the data of Frandon et al., obtained from electron energy loss spectroscopy on $\mathrm{SrF}_{2}$ films.

Fig. 7 displays $\mathrm{k}$ in the spectral range around the F K edge; data calculated with the independent-atom approximation are also shown for comparison. The shape and position of the $\mathrm{F} \mathrm{K}$ edge may be useful for spectroscopy applications. The oscillation above this edge might be attributed to X-ray Absorption

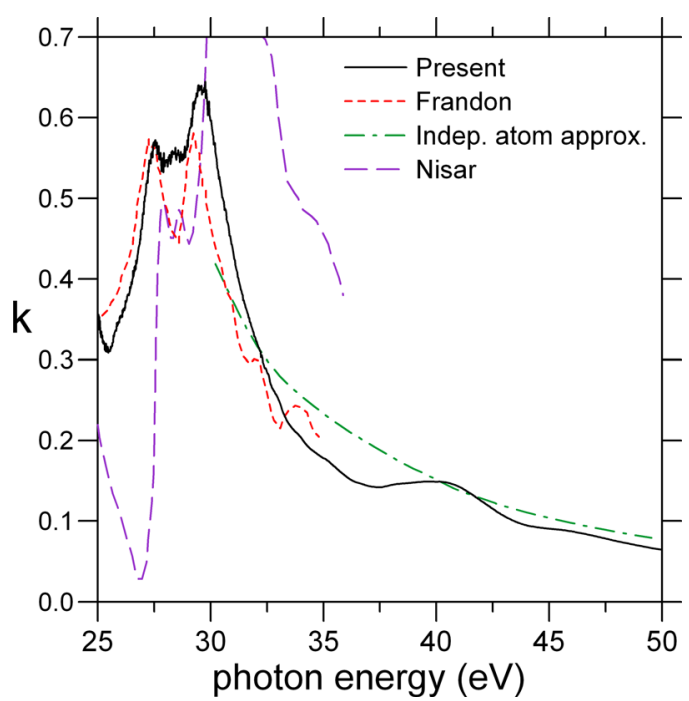

FIG. 6. $\mathrm{k}$ of $\mathrm{SrF}_{2}$ films at low photon energies. The data of Frandon et al. ${ }^{4}$ and the data calculated in the independent-atom approximation are also plotted. The data of Nisar and Robin, ${ }^{1}$ which were measured on single crystals, are also displayed
Fine Structure (XAFS). The peaks at $\sim 540 \mathrm{eV}$ are attributed to the $\mathrm{O} \mathrm{K}$ edge, and hence to the presence of some oxygen in the films. A peak near $540 \mathrm{eV}$ is also present in the calculation with the independent-atom approximation, probably again due to the presence of some oxygen in the Sr films that were used to obtain the optical constants of $\mathrm{Sr}^{16}$

\section{B. Refractive index calculation through dispersion relations}

$\mathrm{n}$, the real part of the refractive index, is calculated with $\mathrm{KK}$ dispersion relations

$$
\mathrm{n}(\mathrm{E})-1=\frac{2}{\pi} \mathrm{P} \int_{0}^{\infty} \frac{\mathrm{E}^{\prime} \mathrm{k}\left(\mathrm{E}^{\prime}\right)}{\mathrm{E}^{\prime 2}-\mathrm{E}^{2}} \mathrm{dE^{ \prime }}
$$

where $\mathrm{P}$ stands for the Cauchy principal value. In order to perform this integration, we need to know $\mathrm{k}$ in the whole spectrum. Hence, we extended our data with data from the literature, along with interpolations, and extrapolations. From the scant literature available on the optical constants of $\mathrm{SrF}_{2}$ films, which was reviewed in Sec. I, the research of Frandon et $a l .{ }^{4}$ provided the largest useful data range and hence it was selected here; we could read their data down to $8 \mathrm{eV}$. At photon energies lower than this, no data were found. This corresponds to the $\mathrm{SrF}_{2}$ range of transparency, at photon energies below the $\mathrm{SrF}_{2}$ cutoff at $\sim 9.8 \mathrm{eV}$. One choice would be to use $\mathrm{k}$ data values obtained from $\mathrm{SrF}_{2}$ crystals. ${ }^{22-24}$ However, in the transparent range extending from the far UV to the far IR, $\mathrm{k}$ for crystalline $\mathrm{SrF}_{2}$ is extremely low, such as $\sim 10^{-6}$ and below. ${ }^{5}$ In contrast, for films deposited at room temperature and in the same range, $\mathrm{k}$ is expected to take much larger values, ${ }^{8,9}$ on the order of $\sim 10^{-2}$; for $\mathrm{MgF}_{2}$ films, this excess absorption was attributed to scattering from inhomogeneities and absorption from the low energy tail of an exciton band. ${ }^{9}$ In order to fill the gap of the $\mathrm{SrF}_{2}$ transparency range, we decided to interpolate between literature data in the adjacent ranges. At photon energies above this transparency gap, we used the data of Frandon et al., ${ }^{4}$

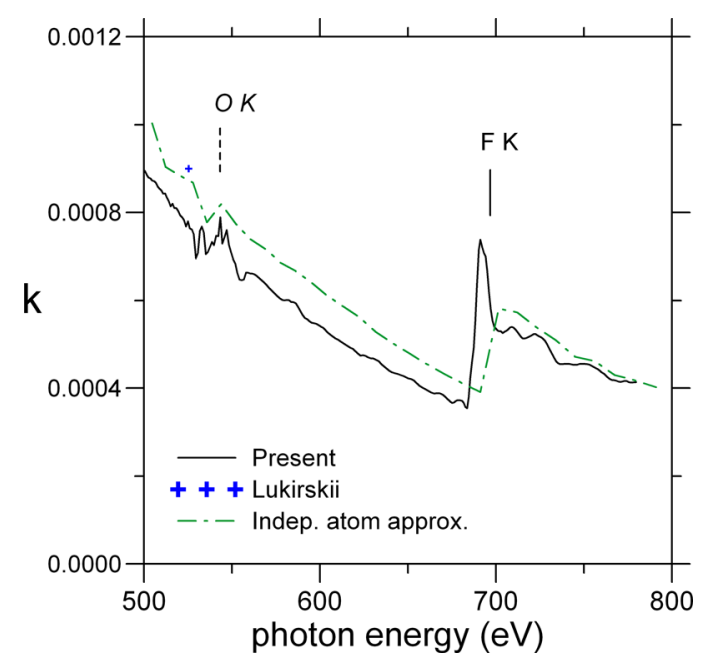

FIG. 7. $\mathrm{k}$ of $\mathrm{SrF}_{2}$ films versus photon energy close to the $\mathrm{F} \mathrm{K}$ edge. One photon energy measured by Lukirskii et $a^{2}{ }^{2}$ and the data calculated in the independent-atom approximation are also plotted. 
which were obtained from $\mathrm{SrF}_{2}$ films. In the far IR, materials with ionic bonding like $\mathrm{SrF}_{2}$ exhibit the reststrahlen band, with strong optical constant variation and large absorption. ${ }^{25,26}$ Since no data on the reststrahlen band was found for $\mathrm{SrF}_{2}$ films in the literature, in the interpolation, we used data on $\mathrm{SrF}_{2}$ crystals by Kaiser et al ${ }^{27}$ and Bosomworth, ${ }^{28}$ as tabulated in Ref. 5. The data of Ref. 4 display several peaks and valleys; we fitted these data with a set of seven Lorentz oscillators to reproduce the main seven peaks of Ref. 4. We added one further oscillator to fit the reststrahlen band. This totaled a fit with eight Lorentz oscillators, which is displayed in Fig. 8. Both Kaiser's data and the fit were somewhat modified in order to obtain a smooth connection, as displayed in Fig. 8. We used Lorentz oscillators because they are complex functions satisfying KK dispersion relations. Additionally, the real part of the sum of the eight Lorentz oscillators used here showed a satisfactory match with the data of Frandon $\mathrm{et} \mathrm{al}^{4}$ and Kaiser $\mathrm{et} \mathrm{al.}^{27}$

In the extrapolation to photon energies larger than $780 \mathrm{eV}$, we used the semiempirical data of Henke et al., ${ }^{19}$ as downloaded from the CXRO's web site,${ }^{20}$ at photon energies up to $3 \times 10^{4} \mathrm{eV}$, and the calculations of Chantler et $\mathrm{al}^{29}$ in the $3 \times 10^{4}$ to $4 \times 10^{5} \mathrm{eV}$ range; in both cases, we used a density of $3.98 \mathrm{~g} / \mathrm{cm}^{3}$ for $\mathrm{SrF}_{2}$. The extrapolation to even larger photon energies was performed by keeping constant the slope of $k(E)$ from Chantler's data in a log-log plot. Fig. 9 displays the whole $\mathrm{k}$ data set gathered in this research.

The data gathered in Fig. 9 was used to obtain $\delta=1-\mathrm{n}$ with Eq. (3); $\delta$ is plotted in Fig. 10. An excellent agreement with the data calculated with the independent-atom approximation was obtained at $\mathrm{E}>45 \mathrm{eV}$. Again, the agreement with the Lukirskii data ${ }^{2}$ is poor. Fig. 11 shows $\mathrm{n}$ at small photon energies.

\section{Consistency of optical constants}

Two sum-rules were used to evaluate the consistency of the above optical constants: the $f$-sum and the inertial sum rule. To apply the $f$-sum rule, it is useful to define the

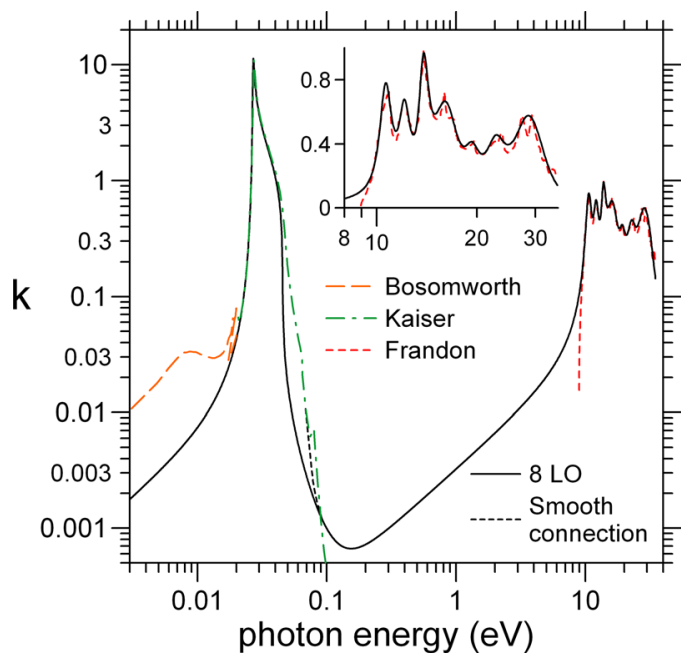

FIG. 8. Log-log plot of $\mathrm{k}$ at low photon energies and the fit with eight Lorentz oscillators to Frandon's data ${ }^{4}$ and to the reststrahlen band, ${ }^{27,28}$ along with a smooth connection to the latter. Inset: $\mathrm{k}$ versus the logarithm of energy showing the fit to Frandon's peaks.

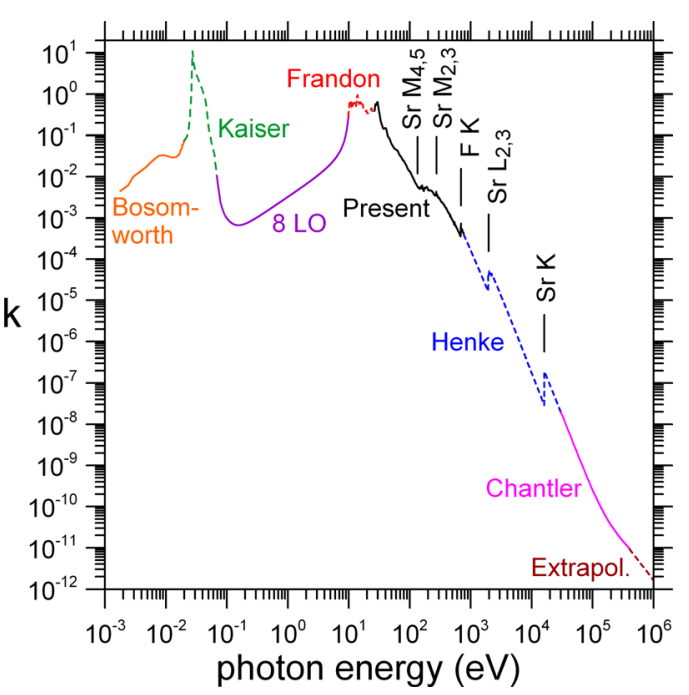

FIG. 9. Log-log plot of k versus photon energy in the whole spectrum gathered in this research.

effective number of electrons per atom $n_{\text {eff }}(E)$ contributing to $k$ up to a given photon energy $E^{30}$

$$
\mathrm{n}_{\mathrm{eff}}(\mathrm{E})=\frac{4 \varepsilon_{0} \mathrm{~m}}{\pi \mathrm{Ne}^{2} \hbar^{2}} \int_{0}^{\mathrm{E}} \mathrm{E}^{\prime} \mathrm{k}\left(\mathrm{E}^{\prime}\right) \mathrm{dE}^{\prime},
$$

where $N$ is the molecule density, which was calculated using the mass density of $3.98 \mathrm{~g} / \mathrm{cm}^{3}, e$ and $m$ are the electron charge and mass, respectively, $\varepsilon_{0}$ is the permittivity of vacuum, and $\hbar$ is the reduced Planck's constant. $f$-sum rule expresses that the high-energy limit of the effective number of electrons must reach the number of electrons in a $\mathrm{SrF}_{2}$ molecule, i.e., 56. When the relativistic correction on scattering factors is taken into account, the high-energy limit of integration with Eq. (4) is somewhat modified. The theoretical effective number of electrons is then reduced to $55.71 .^{31}$ By integrating $\mathrm{k}$ data gathered in the whole spectral range plotted in Fig. 9, we got 55.71, which is exactly the

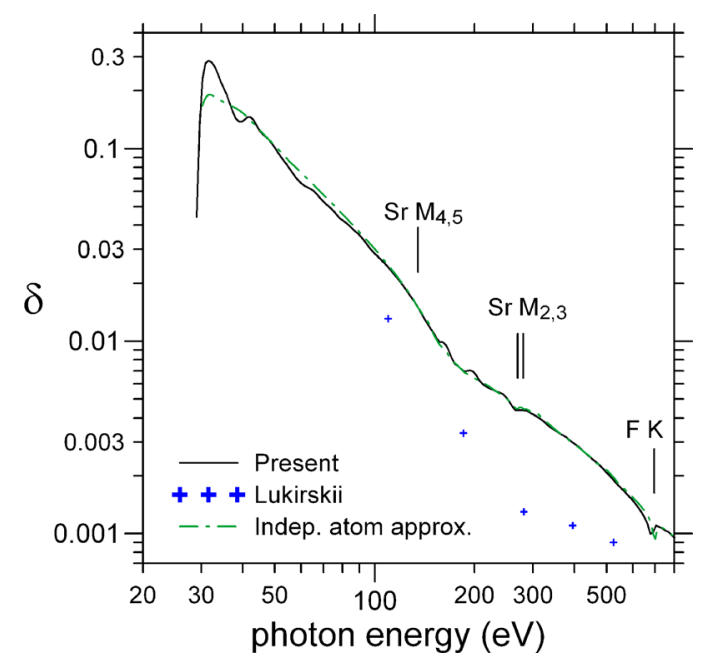

FIG. 10. Log-log plot of $\delta=1-\mathrm{n}$ of $\mathrm{SrF}_{2}$ films versus photon energy. The data of Lukirskii et al. $^{2}$ and the data calculated in the independent-atom approximation are also plotted. 


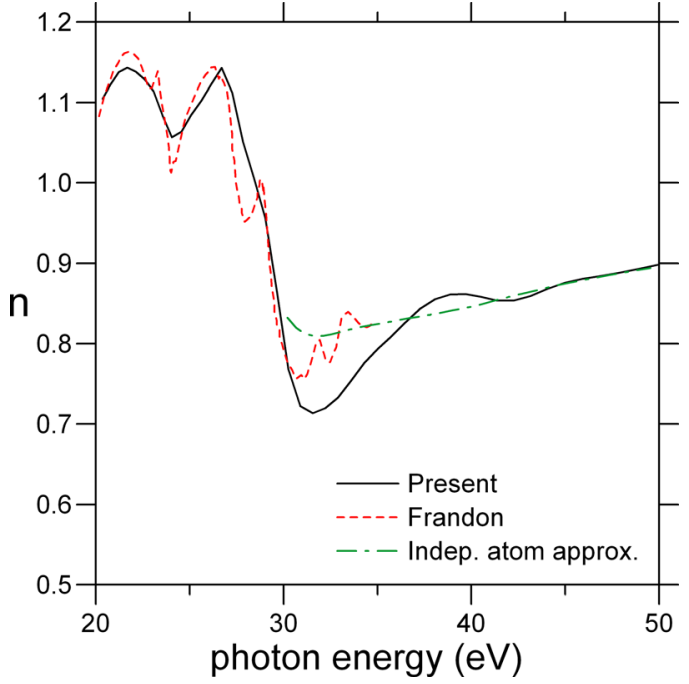

FIG. 11. $\mathrm{n}$ of $\mathrm{SrF}_{2}$ films at low photon energies. The data of Frandon et al. ${ }^{4}$ and the data calculated in the independent-atom approximation are also plotted.

theoretical number. This exact agreement may contain a fortuitous component, and in fact any difference within $\sim \pm 3 \%$ would have been considered satisfactory. The main contribution to the integral was found to come from the $\sim 10-2 \times 10^{4} \mathrm{eV}$ range. The range measured in this research amounted for $\sim 50 \%$ of the total effective number of electrons.

The inertial sum rule is given by

$$
\int_{0}^{\infty}[n(E)-1] d E=0 .
$$

It expresses that the average refractive index over the spectrum must be unity. In order to apply the inertial sum rule, the following normalization parameter of the above integral was used: ${ }^{30}$

$$
\zeta=\frac{\int_{0}^{\infty}[n(E)-1] d E}{\int_{0}^{\infty}|n(E)-1| d E},
$$

Shiles et al. ${ }^{32}$ proposed that a good value of $\zeta$ should stand within \pm 0.005 . An evaluation parameter of $\zeta=-1.3 \times 10^{-4}$ was obtained with the $n$ data set obtained in this research. As with the $f$-sum rule, the evaluation parameter for the inertial sum rule resulted in an excellent value, which suggests a good consistency of the present optical constants of $\mathrm{SrF}_{2}$.

The data are available on request at the following e-mail address: larruquert@io.cfmac.csic.es.

\section{CONCLUSIONS}

The transmittance of $\mathrm{SrF}_{2}$ thin films has been measured for the first time in a large portion of the EUV and SXR ranges. The transmittance measurements were used to calculate the film density, from which it was observed that the density decreased with increasing film thickness. The extinction coefficient was obtained from the linear dependence of the logarithm of transmittance versus the product of thickness and density. Noteworthy features of these data include the $\mathrm{F} \mathrm{K}$ absorption edge and the $\mathrm{Sr} \mathrm{M}_{2,3}$ and $\mathrm{M}_{4,5}$ absorption edges fine structures. The $\mathrm{SrF}_{2}$ refractive index was obtained with the Kramers-Krönig analysis of the extinction coefficient $(\mathrm{k})$ data, which were extended to a larger spectrum using literature data, interpolations, and extrapolations. A lack of data for $\mathrm{SrF}_{2}$ films in the transparent region of $\mathrm{SrF}_{2}$ was filled with a fit involving the combination of eight Lorentz oscillators. The consistency of the optical constants was found to be excellent with the use of $f$ and inertial sum rules. The optical constants of $\mathrm{SrF}_{2}$ films obtained herein in the $25-780 \mathrm{eV}$ range are the first reported experimental data above $\sim 35 \mathrm{eV}$, except for earlier data at five individual photon energies (Ref. 2).

\section{ACKNOWLEDGMENTS}

This work was supported in part by the National Programme for Space Research, Subdirección General de Proyectos de Investigación, Ministerio de Economía y Competitividad, Project No. AYA2010-22032. This work was performed in part under the auspices of the U.S. Department of Energy by Lawrence Livermore National Laboratory under Contract No. DE-AC52-07NA27344 and the University of California Lawrence Berkeley National Laboratory under Contract No. DE-AC03-76F00098. The Advanced Light Source was supported by the Director, Office of Science, Office of Basic Energy Sciences, of the U.S. Department of Energy under Contract No. DE-AC0205CH11231. L. Rodríguez-de Marcos is thankful to Consejo Superior de Investigaciones Científicas (CSIC) for funding under Programa J.A.E. (Junta de Ampliación de Estudios), which was partially supported by the European Social Fund. The technical assistance of J. M. Sánchez is acknowledged.

\footnotetext{
${ }^{1}$ M. Nisar and S. Robin, Pak J. Sci. Ind. Res. 17, 49 (1974).

${ }^{2}$ A. P. Lukirskii, E. P. Savinov, O. A. Ershov, and Yu. F. Shepelev, Opt. Spectrosc. 16, 168 (1964).

${ }^{3}$ S. Robin-Kandare and J. Robin, C. R. Acad. Sci. Paris 262, 1020 (1966).

${ }^{4}$ J. Frandon, B. Lahaye, and F. Pradal, Phys. Status Solidi B 53, 565 (1972).

${ }^{5}$ M. E. Thomas, "Strontium fluoride $\left(\mathrm{SrF}_{2}\right)$," in Handbook of Optical Constants of Solids III, edited by E. D. Palik (Academic Press, San Diego, 1998).

${ }^{6}$ U. Kaiser, N. Kaiser, P. Weilßbrodt, U. Mademann, E. Hacker, and H. Müller, Thin Solid Films 217, 7 (1992).

${ }^{7}$ N. Kaiser, Optical Interference Coatings, Springer Series in Optical Sciences, edited by N. Kaiser and H. K. Pulker (Springer-Verlag, Berlin, 2003).

${ }^{8}$ G. Hass and J. B. Ramsey, Appl. Opt. 8, 1115 (1969).

${ }^{9}$ O. R. Wood II, H. G. Craighead, J. E. Sweeney, and P. J. Maloney, Appl. Opt. 23, 3644 (1984).

${ }^{10}$ A. V. Valeev, Ph.D. dissertation, Kazan University, 1965, as cited by Gisin in Ref. 11.

${ }^{11}$ M. A. Gisin, Opt. Spectrosc. 27, 194 (1969).

${ }^{12}$ A. A. El-Shazly and M. H. Ebrahim, Appl. Phys. 22, 107 (1980).

${ }^{13}$ S. Tolansky, Multiple-Beam Interferometry of Surfaces and Films (Oxford University Press, London, 1948).

${ }^{14}$ J. H. Underwood and E. M. Gullikson, J. Electron Spectrosc. Relat. Phenom. 92, 265 (1998).
} 
${ }^{15}$ E. M. Gullikson, S. Mrowka, and B. B. Kaufmann, Proc. SPIE 4343, 363 (2001).

${ }^{16}$ L. Rodríguez-de Marcos, J. I. Larruquert, J. A. Aznárez, M. Vidal-Dasilva, S. García-Cortés, J. A. Méndez, L. Poletto, F. Frassetto, A. M. Malvezzi, D. Bajoni, A. Giglia, N. Mahne, and S. Nannarone, J. Appl. Phys. 111, 113533 (2012).

${ }^{17}$ D. L. Windt, Comput. Phys. 12, 360-370 (1998) available at http://www.rxollc.com/idl/index.html.

${ }^{18}$ E. M. Gullikson, "Optical properties of materials," in Vacuum Ultraviolet Spectroscopy I, edited by J. A. Samson and D. L. Ederer (Academic Press, San Diego, CA, 2000).

${ }^{19}$ B. L. Henke, E. M. Gullikson, and J. C. Davis, At. Data Nucl. Data Tables 54, 181 (1993).

${ }^{20}$ See http://henke.lbl.gov/optical_constants/ for semi-empirical optical constants of fluorine.

${ }^{21}$ B. R. Benware, M. Seminario, A. L. Lecher, J. J. Rocca, Yu. A. Unspenskii, A. V. Vinogradov, V. V. Kondratenko, Yu. P. Pershing, and B. Bach, J. Opt. Soc. Am. B 18, 1041 (2001).

${ }^{22}$ T. Tomiki and T. Miyata, J. Phys. Soc. Jpn. 27, 658 (1969).

${ }^{23}$ A. Hordvik and L. Skolnik, Appl. Opt. 16, 2919 (1977).
${ }^{24}$ J. A. Harrington, B. L. Bobbs, M. Braunstein, R. K. Kim, R. Stearns, and R. Braunstein, Appl. Opt. 17, 1541 (1978).

${ }^{25}$ S. K. Andersson and C. G. Ribbing, Proc. SPIE 2253, 1042 (1994).

${ }^{26}$ S. Zhao and C.-G. Ribbing, Chin. Opt. Lett. 8, 119 (2010).

${ }^{27}$ W. Kaiser, W. G. Spitzer, R. H. Kaiser, and L. E. Howarth, Phys. Rev. 127, 1950 (1962).

${ }^{28}$ D. R. Bosomworth, Phys. Rev. 157, 709 (1967).

${ }^{29}$ C. T. Chantler, K. Olsen, R. A. Dragoset, J. Chang, A. R. Kishore, S. A. Kotochigova, and D. S. Zucker, X-Ray form Factor, Attenuation, and Scattering Tables (version 2.1) (National Institute of Standards and Technology, Gaithersburg, MD, 2005), available online: http://physics. nist.gov/ffast (29 May 2006); C. T. Chantler, J. Phys. Chem. Ref. Data 29, 597-1048 (2000); 24, 71-643 (1995).

${ }^{30}$ M. Altarelli and D. Y. Smith, Phys. Rev. B 9, 1290 (1974).

${ }^{31}$ Downloaded from the following web of Physical Reference Data, Physics Laboratory at NIST: http://physics.nist.gov/PhysRefData/FFast/Text/ cover.html.

${ }^{32}$ E. Shiles, T. Sasaki, M. Inokuti, and D. Y. Smith, Phys. Rev. B 22, 1612 (1980). 